\title{
Nanoscale tracking of oxygen diffusion pathways in oxide ion conductors
}

David Diercks $^{1}$, Federico Baiutti ${ }^{2}$, Francesco Chiabrera $^{2}$, Alex Morata $^{2}$ and Albert Tarancon ${ }^{2}$

${ }^{1}$ Colorado School of Mines, Golden, Colorado, United States, ${ }^{2}$ Catalonia Institute for Energy Research (IREC), United States

Solid oxide cells which convert chemical energy to electrical energy offer a possible technology for both near and long term efficient and clean energy generation systems [1, 2]. In particular, mixed ionicelectronic conducting oxide (MIEC)-based ceramics, which combine electronic and ionic conductivity, exhibit a promising combination of catalytic and transport behavior for both stationary and portable power generation. [3]. The engineering of the electrochemical properties of MIEC materials at the grain boundaries and phase boundaries has shown great potential [4-6]. In order to improve upon the design of such materials, a better understanding of the nanoscale structure and especially the direct analysis of how the boundaries affect oxygen transport is valuable.

Atom probe tomography (APT) has the unique ability to probe the nanoscale 3-D distribution of species down to tens of parts-per-million concentrations. This has provided fundamental insights into the compositions and composition profiles in and around precipitates, grain boundaries, phase boundaries, and dislocations in metal alloys, electronic materials, and ceramic materials [7]. Additionally, since this time-of-flight technique has sufficient mass resolution to detect particular isotopes of species, as shown in Figure 1a, the introduction of tracer isotopic species can be used to distinguish sources of an element. For example, this technique has been used to investigate hydrogen embrittlement of steels using a deuterium tracer [8].

Here, annealing of lanthanum manganite and chromite films and multiphase MIEC structures at controlled temperatures and times was carried out in an atmosphere containing an ${ }^{18} \mathrm{O}$ isotopic tracer followed by APT analysis of these materials. From the measured distribution of the ${ }^{18} \mathrm{O}$ species, the diffusion of reactant oxygen is distinguished from that in the original oxide structures allowing fundamental investigations on oxygen kinetics at the nanoscale. As exemplified in Figure 1b, the local distribution of ${ }^{18} \mathrm{O}$ (and other species) throughout the MIEC materials are visualized and quantified. This work presents a potentially important tool for assessing the nanoscale transport behavior in oxide ion conducting materials [9].

\section{References}

[1] E.D. Wachsman, K.T. Lee, Lowering the Temperature of Solid Oxide Fuel Cells, Science, 334 (2011) 935-939.

[2] S.S. Shin, J.H. Kim, K.T. Bae, K.-T. Lee, S.M. Kim, J.-W. Son, M. Choi, H. Kim, Multiscale structured low-temperature solid oxide fuel cells with $13 \mathrm{~W}$ power at $500{ }^{\circ} \mathrm{C}$, Energy \& Environmental Science, 13 (2020) 3459-3468.

[3] Z. Gao, L.V. Mogni, E.C. Miller, J.G. Railsback, S.A. Barnett, A perspective on low-temperature solid oxide fuel cells, Energy \& Environmental Science, 9 (2016) 1602-1644. 

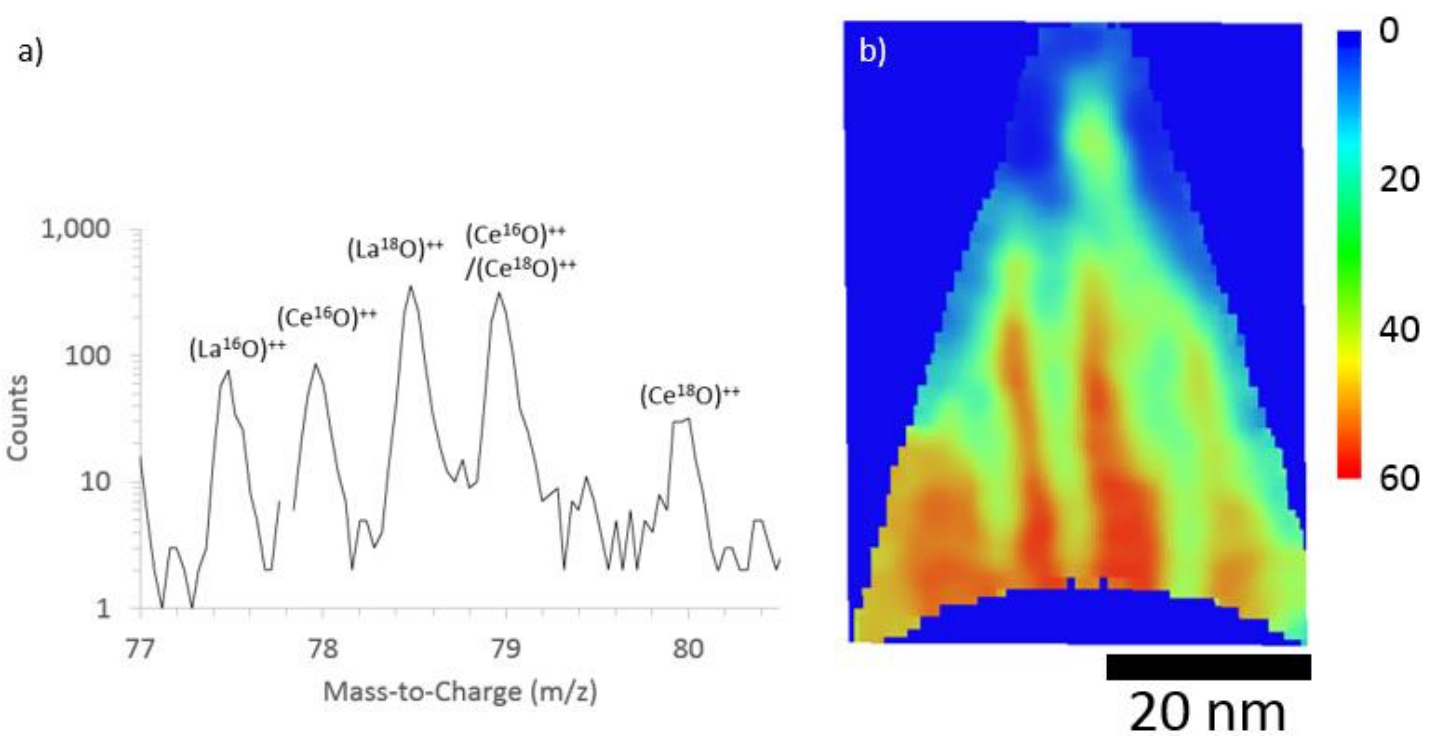

Figure 1. a) Portion of the APT mass spectrum from an MIEC material showing clear resolution of peaks related to the different oxygen isotopes. b) Slice through the APT reconstruction showing the atomic percent distribution of 180 .

[4] E. Navickas, T.M. Huber, Y. Chen, W. Hetaba, G. Holzlechner, G. Rupp, M. Stöger-Pollach, G. Friedbacher, H. Hutter, B. Yildiz, J. Fleig, Fast oxygen exchange and diffusion kinetics of grain boundaries in Sr-doped LaMnO3 thin films, Physical Chemistry Chemical Physics, 17 (2015) 7659-7669. [5] F. Chiabrera, I. Garbayo, L. López-Conesa, G. Martín, A. Ruiz-Caridad, M. Walls, L. Ruiz-González, A. Kordatos, M. Núñez, A. Morata, S. Estradé, A. Chroneos, F. Peiró, A. Tarancón, Engineering Transport in Manganites by Tuning Local Nonstoichiometry in Grain Boundaries, Advanced Materials, 31 (2019) 1805360 .

[6] M. Acosta, F. Baiutti, A. Tarancón, J.L. MacManus-Driscoll, Nanostructured Materials and Interfaces for Advanced Ionic Electronic Conducting Oxides, Advanced Materials Interfaces, 6 (2019) 1900462.

[7] A. Devaraj, D.E. Perea, J. Liu, L.M. Gordon, T.J. Prosa, P. Parikh, D.R. Diercks, S. Meher, R.P. Kolli, Y.S. Meng, S. Thevuthasan, Three-dimensional nanoscale characterisation of materials by atom probe tomography, International Materials Reviews, (2017) 1-34.

[8] Y.S. Chen, D. Haley, S.S.A. Gerstl, A.J. London, F. Sweeney, R.A. Wepf, W.M. Rainforth, P.A.J. Bagot, M.P. Moody, Direct observation of individual hydrogen atoms at trapping sites in a ferritic steel, Science, 355 (2017) 1196.

[9] Portions of this research were supported through the National Institute of Standards and Technology Award 70NANB18H275. The Raman imaging - SEM/FIB instrument used for some of the specimen preparation was acquired through the support of the National Science Foundation (DMR-1828454). 